\title{
A Semiclassical Approach to Level Crossing in Supersymmetric Quantum Mechanics
}

\author{
J. F. Beacom * and A. B. Balantekin ** \\ Department of Physics, University of Wisconsin, Madison, WI 53706, USA
}

\begin{abstract}
Much use has been made of the techniques of supersymmetric quantum mechanics (SUSY QM) for studying bound-state problems characterized by a superpotential $\varphi(x)$. Under the analytic continuation $\varphi(x) \rightarrow i \varphi(x)$, a pair of superpartner bound-state problems is transformed into a two-state level-crossing problem in the continuum. The description of matter-enhanced neutrino flavor oscillations involves a level-crossing problem. We treat this with the techniques of supersymmetric quantum mechanics. For the benefit of those not familiar with neutrino oscillations and their description, enough details are given to make the rest of the paper understandable. Many other level-crossing problems in physics are of exactly the same form. Particular attention is given to the fact that different semiclassical techniques yield different results. The best result is obtained with a uniform approximation that explicitly recognizes the supersymmetric nature of the system.
\end{abstract}

\section{SUSY QM and the Bound-State Problem}

Starting with a superpotential $\varphi(x)$, one can generate two superpartner potentials

$$
V_{ \pm}=\varphi^{2}(x) \pm \frac{\hbar}{\sqrt{2 m}} \varphi^{\prime}(x) .
$$

For these two potentials the two corresponding Schrödinger equations are

$$
\left[-\frac{\hbar^{2}}{2 m} \frac{\partial^{2}}{\partial^{2} x}+V_{ \pm}\right] \Psi_{ \pm}(x)=E \Psi_{ \pm}(x) .
$$

It can be shown that the eigenspectrum of the "+" system can be obtained by shifting the quantum numbers $n$ of the "-" system by $n \rightarrow n-1$, with the ground state of the "-" system discarded. That is, the spectra of the two systems are identical except for a single state. In applications, this property is exploited in the following way. Given a potential $V(x)$, one attempts to find a superpotential $\varphi(x)$ that will generate $V(x)$ via Eq. (1), with one or the other sign. If this can be done, one can immediately generate the superpartner potential by choosing the opposite sign in Eq. (1). In some fortunate circumstances, the equations of motion for the second system are much easier to solve than the first. See Schwabl

\footnotetext{
* Speaker. Current address: Physics 161-33, Caltech, Pasadena, CA 91125, USA.

beacom@citnp.caltech.edu

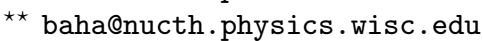


(1995) for more introductory material, and Cooper et al. (1995) and references therein for active areas of research.

The application of supersymmetric quantum mechanics to the solution of bound-state problems has been extensively developed. There has been particular interest in semiclassical techniques. A direct primitive semiclassical (WKB) approach to the Schrödinger equation yields the usual Bohr-Sommerfeld quantization condition:

$$
\sqrt{2 m} \int_{x_{1}}^{x_{2}} d x \sqrt{E-V(x)}=\left(n+\frac{1}{2}\right) \hbar \pi,
$$

where $x_{1}$ and $x_{2}$ are the turning points (zeros of the integrand). Since the superpartner potentials depend explicitly on $\hbar$, the Schrödinger equations in Eq. (2) have a different dependence on $\hbar$ than the usual case. A primitive semiclassical (WKB) solution of Eq. (2), which however explicitly recognizes the supersymmetric nature of the system [Comtet et al. (1985)], therefore yields a modified quantization condition:

$$
\sqrt{2 m} \int_{x_{1}}^{x_{2}} d x \sqrt{E-\phi^{2}(x)}=n \hbar \pi,
$$

where $x_{1}$ and $x_{2}$ are the turning points (zeros of the integrand). This modified quantization condition is exact for many systems (see Hruska et al. (1997) for a catalog of results), but not all [DeLaney and Nieto (1990)]. The WKB wave functions are singular at each turning point. It is possible to avoid this problem by using a uniform approximation - one that is valid for all $x$, including at the turning points. The approach is similar to WKB, but there is a special construction to cancel the turning-point singularity. A uniform semiclassical solution [Fricke et al. (1988)] of Eq. (2) recovers the modified quantization condition. However, it gives a much better wave function, which will be essential for the transition probability derived below.

\section{Introduction to Neutrino Oscillations}

\subsection{Vacuum Neutrino Oscillations}

It is possible that the flavor and mass eigenstates of neutrinos are not identical. Throughout this paper, we consider mixing between only two flavors, electron and muon. Then a general state can be written in the flavor basis:

$$
|\nu(t)\rangle=\Psi_{e}(t)\left|\nu_{e}\right\rangle+\Psi_{\mu}(t)\left|\nu_{\mu}\right\rangle
$$

or the mass basis:

$$
|\nu(t)\rangle=\Psi_{1}(t)\left|\nu_{1}\right\rangle+\Psi_{2}(t)\left|\nu_{2}\right\rangle .
$$

The amplitudes are defined as

$$
\Psi_{e}\left(\Psi_{\mu}\right)=\text { amplitude for the neutrino to have flavor } e(\mu)
$$


$\Psi_{1}\left(\Psi_{2}\right)=$ amplitude for the neutrino to have mass $m_{1}\left(m_{2}\right)$.

The amplitudes are taken to be time-dependent, and the kets to be time-independent. For two flavors, the flavor and mass bases must be related by a simple rotation. This rotation is taken to be between the amplitudes, with the kets held fixed, and is given by:

$$
\left[\begin{array}{l}
\Psi_{1}(t) \\
\Psi_{2}(t)
\end{array}\right]=\left[\begin{array}{cc}
\cos \theta_{v} & -\sin \theta_{v} \\
\sin \theta_{v} & \cos \theta_{v}
\end{array}\right]\left[\begin{array}{l}
\Psi_{e}(t) \\
\Psi_{\mu}(t)
\end{array}\right],
$$

where $\theta_{v}$ is the vacuum mixing angle.

In the mass basis, the Schrödinger equation is

$$
i \hbar \frac{\partial}{\partial t}\left[\begin{array}{c}
\Psi_{1}(t) \\
\Psi_{2}(t)
\end{array}\right]=H_{\text {mass }}\left[\begin{array}{c}
\Psi_{1}(t) \\
\Psi_{2}(t)
\end{array}\right]
$$

where

$$
H_{\text {mass }}=\left[\begin{array}{cc}
E_{1} & 0 \\
0 & E_{2}
\end{array}\right],
$$

and is diagonal by definition. Since the neutrino masses $m_{1}$ and $m_{2}$ are presumed small, we make an ultrarelativistic expansion (using $c=1$ units here and below). Defining $E$ to be the common energy, and defining the mass-squared difference as

$$
\delta m^{2}=m_{2}^{2}-m_{1}^{2},
$$

one can show that $H_{\text {mass }}$ is given by

$$
H_{\text {mass }}=\left(E+\frac{m_{1}^{2}+m_{2}^{2}}{4 E}\right)\left[\begin{array}{ll}
1 & 0 \\
0 & 1
\end{array}\right]+\frac{\delta m^{2}}{4 E}\left[\begin{array}{rr}
-1 & 0 \\
0 & 1
\end{array}\right] .
$$

Below, the term proportional to the identity matrix will be dropped.

Now we change to the flavor basis, using the rotation matrix above that relates the two bases. In the flavor basis, the Schrödinger equation is

$$
i \hbar \frac{\partial}{\partial t}\left[\begin{array}{c}
\Psi_{e}(t) \\
\Psi_{\mu}(t)
\end{array}\right]=H_{\text {flavor }}\left[\begin{array}{l}
\Psi_{e}(t) \\
\Psi_{\mu}(t)
\end{array}\right],
$$

where

$$
H_{\text {flavor }}=\frac{\delta m^{2}}{4 E}\left[\begin{array}{cc}
-\cos 2 \theta_{v} & \sin 2 \theta_{v} \\
\sin 2 \theta_{v} & \cos 2 \theta_{v}
\end{array}\right] .
$$

Using the rotation matrix, the amplitude to be of the electron type at a time $t$ is

$$
\Psi_{e}(t)=\cos \theta_{v} \Psi_{1}(t)+\sin \theta_{v} \Psi_{2}(t) .
$$


The time evolution of the mass amplitudes is trivial, so that just a phase relates the amplitudes at a point $t$ to those at the point $t=0$. The mass amplitudes at $t=0$ can be expressed in terms of the flavor amplitudes at $t=0$ by use of the rotation matrix. Taking as initial conditions $\Psi_{e}(0)=1, \Psi_{\mu}(0)=0$ (an electron-type neutrino produced at $t=0$ ),

$$
\Psi_{e}(t)=\cos ^{2} \theta_{v} \exp \left(+i \frac{\delta m^{2}}{4 E} \frac{t}{\hbar}\right)+\sin ^{2} \theta_{v} \exp \left(-i \frac{\delta m^{2}}{4 E} \frac{t}{\hbar}\right) .
$$

The probability for the neutrino to be of the electron type can then be shown to be

$$
P\left(\nu_{e} \rightarrow \nu_{e}\right)(t)=\left|\Psi_{e}(t)\right|^{2}=1-\sin ^{2} 2 \theta_{v} \sin ^{2}\left(\pi t / L_{\mathrm{osc}}\right),
$$

where the oscillation length is $L_{\mathrm{osc}}=4 \pi E \hbar / \delta m^{2}$, so called since this is the separation between extrema in the survival probability. Since the initial flavor state was not a stationary state of the Hamiltonian, the probability to be of either flavor oscillates. Note that if either the vacuum mixing angle or the masssquared splitting is small, the effects of the oscillations are minimal. If either the source or detector has a finite size of order $L_{\text {osc }}$ or greater, then after averaging over that region,

$$
\left\langle P\left(\nu_{e} \rightarrow \nu_{e}\right)\right\rangle_{\mathrm{avg}}=1-\frac{1}{2} \sin ^{2} 2 \theta_{v},
$$

which is independent of energy.

\subsection{Matter-Enhanced Neutrino Oscillations}

In this section we assume the vacuum oscillations discussed above. Here we consider that the neutrino is traveling through a medium with a varying density of electrons, e.g., the sun. We consider the scattering

$$
\nu_{x}+e^{-} \rightarrow \nu_{x}+e^{-},
$$

where $\nu_{x}$ indicates either flavor. In such reactions, a neutrino of a given flavor enters and a neutrino of the same flavor leaves. Electron neutrinos undergo both charged- and neutral-current reactions with the electrons, but muon neutrinos undergo only neutral-current scattering. For elastic forward scattering, the coherence of the neutrino "beam" can be maintained. The effect of the medium is to modify the dispersion relation (refractive index) of the neutrino. Equivalently, one can say that the neutrino masses are modified in the medium, and that the modification is different for electron and muon neutrinos. Below, we neglect a term proportional to the identity, and consider only the difference between the electron and muon neutrinos. Then the Hamiltonian in Eq.(13) becomes

$$
H_{\text {flavor }}=\frac{\delta m^{2}}{4 E}\left[\begin{array}{cc}
\zeta(t)-\cos 2 \theta_{v} & \sin 2 \theta_{v} \\
\sin 2 \theta_{v} & -\zeta(t)+\cos 2 \theta_{v}
\end{array}\right],
$$


where $\zeta(t)$ is related to the electron number density $N_{e}(t)$ by

$$
\zeta(t)=\frac{2 \sqrt{2} G_{F} N_{e}(t)}{\delta m^{2} / E}
$$

and $G_{F}$ is the Fermi coupling constant.

When $\zeta(t) \rightarrow 0$, the vacuum mixing case is recovered. When $\zeta(t) \rightarrow \infty$, the Hamiltonian is also considerably simplified. However, when $\zeta(t)=\cos 2 \theta_{v}$, the effect of the off-diagonal coupling is maximal. This is known as the resonance point. In a medium with a varying density, such as the sun, it is possible for an electron neutrino to be produced at a density above the resonance density. Then during its passage out of the sun it will pass through the resonance density. That has profound consequences for the probability that it emerges in vacuum as an electron neutrino.

The flavor-basis Hamiltonian can be instantaneously diagonalized with a rotation matrix, the angle of which is called the matter angle $\theta(t)$. The bases are related as:

$$
\left[\begin{array}{c}
\Psi_{1}(t) \\
\Psi_{2}(t)
\end{array}\right]=\left[\begin{array}{cc}
\cos \theta(t) & -\sin \theta(t) \\
\sin \theta(t) & \cos \theta(t)
\end{array}\right]\left[\begin{array}{c}
\Psi_{e}(t) \\
\Psi_{\mu}(t)
\end{array}\right] .
$$

As the density varies, so does the matter angle. At high densities, the matter angle $\theta \rightarrow \pi / 2$; at resonance, $\theta=\pi / 4$; and at low densities, $\theta \rightarrow \theta_{v}$, the vacuum mixing angle (this is explained in Balantekin and Beacom (1996)).

The instantaneous eigenvalues of the flavor-basis Hamiltonian are (reintroducing the term proportional to the identity matrix):

$$
E+\frac{m_{1}^{2}+m_{2}^{2}+\delta m^{2} \zeta(t)}{4 E} \mp \frac{\delta m^{2}}{4 E} \sqrt{\sin ^{2} 2 \theta_{v}+\left(\zeta(t)-\cos 2 \theta_{v}\right)^{2}} .
$$

If the density changes slowly (adiabatically), then the instantaneous diagonalization will be almost exact and the mass eigenstates will be almost stationary states. (Note that the Hamiltonian is exactly diagonal in the mass basis for any constant density.) If the density changes quickly (nonadiabatically), then the neutrino can "hop" from one eigenstate to another in the neighborhood of the resonance (where the splitting between mass eigenvalues is at a minimum). The probability of this occurring is called the hopping probability $P_{\text {hop }}$. This occurs proportionally to the extent that the instantaneous diagonalization fails. The instantaneous eigenvalues of $H_{\text {flavor }}$ as a function of density are shown schematically in Fig. 1.

Consider an electron neutrino created at a high density and a time $t=0$. Since the matter angle will be near $\pi / 2$, then $\Psi_{e}(0) \approx \Psi_{2}(0)$. If the density changes slowly, the neutrino will remain in this eigenstate. Then, if the vacuum mixing angle is fairly small, then $\Psi_{2}(t) \approx \Psi_{\mu}(t)$ in vacuum. In this case, the electron neutrino created in the solar core will emerge as a muon neutrino, and it will be missed by experiments counting electron neutrinos from the sun. On the other hand, if the density changes rapidly the neutrino may emerge in the 


\section{Instantaneous eigenvalues of $\mathrm{H}_{\text {flavor }}$}

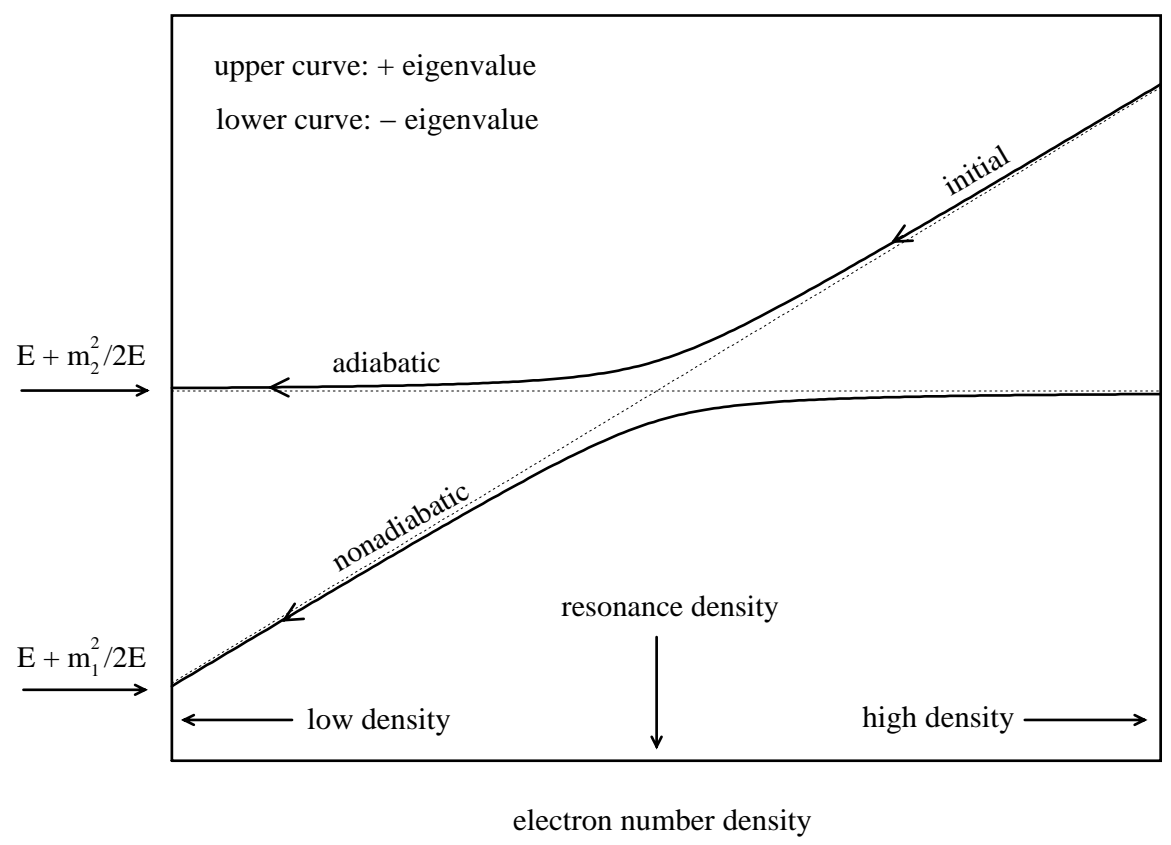

Fig. 1. A schematic illustration of how the instantaneous energy eigenvalues of $H_{\text {flavor }}$ vary as a function of electron number density. The details depend upon the mixing parameters, the energy, and the density profile. Beyond the left edge of the figure, the density is assumed to vanish; the eigenvalues are constant in vacuum and have the values indicated. In the figure, the initial state is assumed to be a mass $=m_{2}$ eigenstate. The final state may be the same eigenstate (adiabatic case) or the other eigenstate (nonadiabatic case). In the figure, the initial and final densities appear to be equally far from the resonance; that is not true in general.

other instantaneous eigenstate. Then, if the vacuum mixing angle is fairly small, then $\Psi_{1}(t) \approx \Psi_{e}(t)$ in vacuum. In this case, the neutrino emerges as an electron neutrino after all.

In a later section, this problem is reformulated so that these extreme limits of the matter and vacuum angle are not necessary. In general then, whether the neutrino emerges from the sun as the electron or muon type is a complicated function of the mixing parameters, the energy, and the form of the density profile. The probability of it emerging as the original flavor is called the survival probability $P\left(\nu_{e} \rightarrow \nu_{e}\right)$; the general form is given in Eq. (31).

For further introductory reading on neutrino oscillations, see Boehm and Vogel (1992). For a recent review of neutrino astrophysics (including oscillations), see Balantekin (1997). 


\section{Supersymmetric Character of the Level-Crossing Problem}

The Hamiltonian of Eq. (19) is a typical form for a level-crossing problem. Away from the resonance point, the off-diagonal elements may be neglected. The diagonal elements approximate the eigenvalues, and are allowed to vary. If the off-diagonal elements were exactly zero, the eigenvalue trajectories would cross (become equal) at the resonance point. This crossing will be avoided if the offdiagonal elements have any nonzero value, no matter how small. In the most general level-crossing problem, the off-diagonal elements would also be allowed to vary. Here they are taken to be constant. Nevertheless, that is not a large practical restriction. Approximating the off-diagonal elements as constant is reasonable as the resonance region (the only region in which they contribute significantly) is usually very narrow. The explicit representation of the supersymmetry in Eq. (19) is given in Balantekin et al. (1988).

Before proceeding further, we switch to working with dimensionless quantities. We define a length scale

$$
L=\frac{\hbar \lambda}{\delta m^{2} / 4 E},
$$

and use this to define $x=t / L$. In Section $1, x$ was used to denote a generic coordinate (with dimensions of length). Here and below, $x$ is dimensionless. Since we will be making a semiclassical expansion, we need to be able to keep track of formal powers of $\hbar$. For each $\hbar$ in the problem, we write $\lambda$ and consider $\lambda$ to be formally small; this is equivalent to saying that the length $L$ is small. We will make expansions in powers of $\lambda$, truncating the higher orders. At the end of the calculation, we will set $\lambda=1$. For notational convenience, we redefine the flavor-basis Hamiltonian as follows:

$$
i \lambda \frac{\partial}{\partial x}\left[\begin{array}{c}
\Psi_{e}(x) \\
\Psi_{\mu}(x)
\end{array}\right]=H_{\text {flavor }}(x)\left[\begin{array}{c}
\Psi_{e}(x) \\
\Psi_{\mu}(x)
\end{array}\right],
$$

where

$$
H_{\text {flavor }}(x)=\left[\begin{array}{cc}
\eta \varphi(x) & \sqrt{\Lambda} \\
\sqrt{\Lambda} & -\eta \varphi(x)
\end{array}\right] \text {. }
$$

We have defined

$$
\eta \varphi(x)=\zeta(x)-\cos 2 \theta_{v}
$$

and

$$
\Lambda=\sin ^{2} 2 \theta_{v} .
$$

(The definition of $\Lambda$ was misprinted in Balantekin and Beacom (1996)). The scaled electron density is

$$
\zeta(x)=\frac{2 \sqrt{2} G_{F} E N_{e}(x)}{\delta m^{2}} .
$$


Note that there are notation changes from previous related works [Fricke et al. (1988), Balantekin et al. (1988), Balantekin and Seger (1991)]; here we have made $\Lambda$ and $\varphi$ dimensionless. The factor $\eta$ (taken to be \pm 1 ), is introduced above to control the analytic behavior of the function $\varphi(x)$ in the complex plane, as explained in Balantekin and Beacom (1996). In the expressions with $\varphi^{2}$ below, we drop $\eta^{2}=1$.

The coupled first-order equations of Eq. (25) can be decoupled to yield

$$
-\lambda^{2} \frac{\partial^{2} \Psi_{e}(x)}{\partial x^{2}}-\left[\Lambda+\varphi^{2}(x)+i \lambda \eta \varphi^{\prime}(x)\right] \Psi_{e}(x)=0
$$

and

$$
-\lambda^{2} \frac{\partial^{2} \Psi_{\mu}(x)}{\partial x^{2}}-\left[\Lambda+\varphi^{2}(x)-i \lambda \eta \varphi^{\prime}(x)\right] \Psi_{\mu}(x)=0,
$$

where $\varphi(x)$ and $\Lambda$ are defined in Eqs. (26) and (27). Such a simple decoupling is not possible in the mass basis. Eqs. (29) and (30) are explicitly of the supersymmetric form. This form follows directly from the (rather general) form of the level-crossing Hamiltonian above. The two levels are superpartners.

These Schrödinger-like equations are similar to those for non-relativistic particles in the presence of a complex barrier, and for convenience we use the language of wave mechanics to describe them. In particular, to the extent that we can ignore the imaginary terms in the potential, these correspond to particles above a barrier (since $\Lambda>0$ ). There are two caveats regarding discussing this as a barrier penetration problem. First, that our boundary conditions do not correspond to the usual picture of incident, reflected, and transmitted waves; in general, there are waves moving in each direction on each side of the barrier. The boundary conditions make it a level-crossing problem instead of a barrier problem. (The condition of a pure electron neutrino at the initial point requires $\Psi_{e}=1, \Psi_{\mu}=0$ there.) Second, the pure imaginary terms in the potentials play an extremely important role here, even in the asymptotic regions. These terms are needed not only to represent nonadiabatic transitions, but also to allow the local matter angle to change. Since our problem is a level-crossing problem, the quantity of interest is not a reflection or transmission coefficient, but rather $P\left(\nu_{e} \rightarrow \nu_{e}\right)=\left|\Psi_{e}(x \rightarrow \infty)\right|^{2}$, the probability of the neutrino being of the electron type far from the source.

\section{Semiclassical Solution of the Equations of Motion}

\subsection{General Form of the Solution}

In general, the survival probability has terms which depend on the source and detector positions. In this paper, these interference terms are considered to be averaged away by the finite source and detector sizes (or by a varying distance between them, such as that due to the motion of the earth). The remaining term below must also be considered to have been appropriately averaged. 
The general form of the survival probability is

$$
P\left(\nu_{e} \rightarrow \nu_{e}\right)=\frac{1}{2}\left[1+\left(1-2 P_{\text {hop }}\right) \cos 2 \theta_{i} \cos 2 \theta_{v}\right] .
$$

The two matter angle terms account for the rotations between the mass and flavor bases at the initial and final (in vacuum) points. (The matter angle thus ranges from $\pi / 2$ at infinite density to $\theta_{v}$ in vacuum. At the resonance, $\theta=\pi / 4$.) $P_{\text {hop }}$ is the probability of hopping from one mass eigenstate to the other during the transit through the resonance region. This result for the survival probability is completely general. The heart of the problem is to determine $P_{\text {hop }}$. Various semiclassical results for $P_{\text {hop }}$ are discussed below. The variation of the averaged survival probability with $\delta \mathrm{m}^{2} / E$ for two choices of the vacuum mixing angle is shown in Fig. 2.

The general shape of the survival probability vs $\delta m^{2} / E$ is that of a pit. Highand low-energy electron neutrinos have a relatively high probability to remain so, whereas medium-energy electron neutrinos have a relatively low probability to remain so (and hence a relatively high probability to transform into muon neutrinos). The solar neutrino detectors are primarily sensitive to electron neutrinos. The energy-dependent suppression of electron neutrinos as in Fig. 2 explains the solar neutrino data well.

\subsection{Primitive Semiclassical Solution}

A primitive semiclassical (WKB) solution can be made to Eqs. (29) and (30). It can be shown that this is exactly equivalent to the adiabatic solution. The adiabatic solution is obtained when the Hamiltonian changes so slowly that the instantaneous diagonalization always holds with high accuracy. That is, there are no transitions between mass eigenstates, so $P_{\text {hop }}=0$ by definition. This result is not shown in Fig. 2. If it were, it would match the exact result in all but the left-hand rise of the pit. Where the other curves rise up, the WKB result would remain flat, at the level of the bottom of the pit.

In the bound-state problem, even the primitive semiclassical solution gave an excellent answer for the quantization condition. As noted, in the neutrino problem this approximation does not predict $P_{h o p}$. The problem comes from the well-known singularity of the WKB wave functions near the turning points. In this problem, the turning points are in the complex plane, near the resonance point (which is on the real axis). In the bound-state problem, the turning point singularity is not as crucial since the quantization condition only counts nodes between the turning points. In this problem, we need the wave function itself to be accurate, since its modulus squared determines the survival probability.

\subsection{Landau-Zener Solution}

Far from the resonance, the propagation is adiabatic, and can be trivially solved for any density profile. A reasonable approach to simplifying the problem is to 
Average survival probability

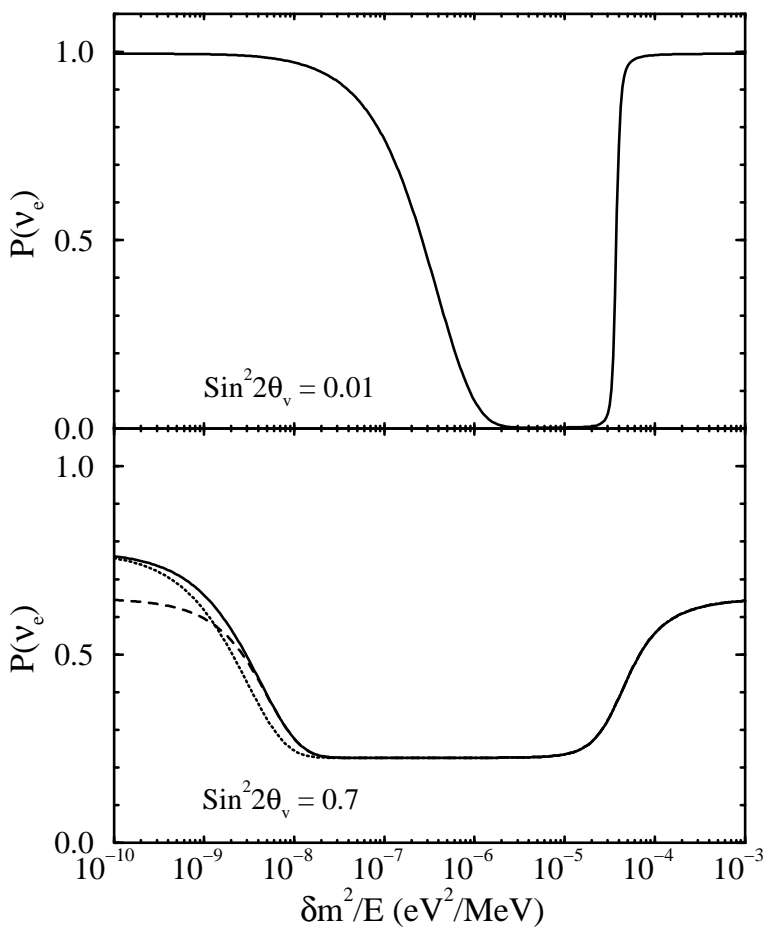

Fig. 2. The electron neutrino survival probability vs. the mass-squared difference parameter for two different vacuum mixing angles. The solid line is given by the method of Balantekin and Beacom (1996). The dashed line is the exact (numerical) result. The dotted line is the linear Landau-Zener result. In the top figure, the lines are indistinguishable. An exponential density with parameters chosen to approximate the sun was used [Bahcall (1989)]. The region leftward of the lower left corner of the trough is the nonadiabatic region.

approximate the density profile as linear in the resonance region. This is the basis of the Landau-Zener solution. In the flavor-basis Hamiltonian of Eq. (25), this gives the usual Landau-Zener setup: linear variation of the diagonal elements, and constant off-diagonal coupling. In order to make Fig. 1 more general, the variation is shown versus the density itself (instead of $x$ ).

The solution is obtained from the decoupled equations (29) and (30). With $\zeta(x) \sim x$, the differential equations can be reduced to the defining equation for the parabolic cylinder (Weber) functions. Given the initial conditions $\Psi_{e}=$ $1, \Psi_{\mu}=0$, the solution for $\Psi_{e}(x)$ at any point is straightforward. (Actually, the boundary conditions require some care since the linear density eventually becomes negative, which is unphysical.) From the asymptotic form appropriate 
far after the resonance, one can extract the hopping probability $P_{\text {hop }}$ from the the survival probability $P\left(\nu_{e} \rightarrow \nu_{e}\right)=\left|\Psi_{e}(x \rightarrow+\infty)\right|^{2}$. The result is

$$
P_{\text {hop }}=\exp \left(-\pi \frac{\delta m^{2}}{4 E \hbar} \frac{\sin ^{2} 2 \theta_{v}}{\cos 2 \theta_{v}}\left|\frac{\dot{\zeta}(t)}{\zeta(t)}\right|_{\text {res }}^{-1}\right) .
$$

Because the Landau-Zener solution is based on the exact solution for the linear density, there are no turning-point singularities as with the WKB solution. Thus the approximate wave function and hence $P_{\text {hop }}$ are fairly reasonable. However, there is some inaccuracy due to the fact that a general density does vary more than linearly through the resonance region. The fact that the LandauZener result has the right general behavior but is not very accurate can be seen in Fig. 2.

\subsection{Uniform Semiclassical Solution}

In this section we outline a uniform semiclassical solution to the problem [Balantekin and Beacom (1996)]. An arbitrary monotonic density profile is allowed, as are nearly arbitrary mixing parameters. We start with Eq. (29):

$$
-\lambda^{2} \frac{\partial^{2} \Psi_{e}(x)}{\partial x^{2}}-\left[\Lambda+\varphi^{2}(x)+i \lambda \eta \varphi^{\prime}(x)\right] \Psi_{e}(x)=0 .
$$

There is no known exact solution of this for arbitrary $\zeta(x)$. Recall that $\lambda$ is a formally small perturbation parameter (we set $\lambda=1$ later), and that $\eta= \pm 1$. Compare this to

$$
-\lambda^{2} \frac{\partial^{2} U(S)}{\partial S^{2}}-\left[\Omega+S^{2} \pm i \lambda \eta\right] U(S)=0,
$$

which is solvable in terms of parabolic cylinder functions. Identification of the turning points of each of these will be crucial. At lowest order in $\lambda$, they are the points for which $\Lambda+\varphi^{2}(x)=0$ and $\Omega+S^{2}=0$, respectively. The turning points are complex conjugate pairs.

Suppose we could find a change of variables $S=S(x)$. Using this, we could deform the simple parabolic "barrier" of the second equation into the more complicated "barrier" shape of the first. Then the solutions of the general case could be expressed in terms of the solutions of the simple case. Such a formal solution can be written as

$$
\Psi_{e}(x)=\frac{1}{\sqrt{S^{\prime}(x)}} U(S(x)) .
$$

Starting with this formal solution, and the two Schrödinger equations above for $\Psi_{e}(x)$ and $U(S)$, one can determine a differential equation for $S=S(x)$. Unsurprisingly, that is a nonlinear equation, and at least as difficult as the original 
Schrödinger equation. However, this equation can be profitably subjected to a semiclassical solution. We expand as

$$
S(x)=S_{0}(x)+\lambda S_{1}(x) \ldots,
$$

and truncate at second order. Separating the various orders in $\lambda$ determines a series of equations. The solution of the $\mathcal{O}(\lambda)$ equation determines $S_{0}(x)$ :

$$
\int_{i \sqrt{\Omega}}^{S_{0}(x)} d S_{0} \sqrt{\Omega+S_{0}^{2}}=\int_{x_{0}}^{x} d t \sqrt{\Lambda+\varphi^{2}(x)}
$$

and also $\Omega$ :

$$
\Omega=\frac{2 i}{\pi} \int_{x_{0}}^{x_{0}^{*}} d x \sqrt{\Lambda+\varphi^{2}(x)},
$$

where $x_{0}$ and $x_{0}^{*}$ are the turning points (zeros of the integrand). The $\mathcal{O}\left(\lambda^{1}\right)$ equation determines $S_{1}(x)$ :

$$
\begin{aligned}
S_{1}(x) & =\frac{i \eta}{2 \sqrt{\Omega+S_{0}^{2}(x)}} \\
& \times\left\{\ln \left[\frac{\varphi+\sqrt{\Lambda+\varphi^{2}(x)}}{\sqrt{\Lambda}}\right]+\ln \left[\frac{\sqrt{\Omega}}{S_{0}(x)+\sqrt{\Omega+S_{0}^{2}(x)}}\right]\right\}
\end{aligned}
$$

The change of variables $S=S(x)$ is actually a mapping of the complex $x$-plane to the complex $S$-plane. In order to avoid spurious branch cut discrepancies, the mapping must be chosen to not fold or flip the plane. This is accomplished with some appropriate choices of signs. Further, the turning points in the $x$-plane must be mapped onto the turning points in the $S$-plane. That demand fixes the definition of $\Omega$ to be the one given above.

The approximate (but uniformly valid) solution is

$$
\Psi_{e}(x) \approx\left[\frac{\Omega+S_{0}^{2}(x)}{\Lambda+\varphi^{2}(x)}\right]^{1 / 4} U\left(S_{0}(x)+\lambda S_{1}(x)\right)
$$

At the turning points, the denominator vanishes, just as in the WKB solution. However, because of the matching of turning points in the mapping, the numerator vanishes at the same points. That cancels the singularity and gives an excellent approximation to the wave function at all points, including near the turning points (and hence near the resonance, which is on the real axis near the turning points).

As noted above, the functions $U(S)$ are parabolic cylinder functions. Using the defining equations for $S_{0}(x)$ and $S_{1}(x)$ given above, one can solve for $\Psi_{e}(x)$ for any $x$. The implicit definition of $S_{0}(x)$ makes it analytically solvable only for large $|x|$ and large $\left|S_{0}\right|$, which holds far from the resonance. However, both the initial and final point can be taken to be far from the resonance. At each point, the general solution can be written in terms of two independent parabolic 
cylinder functions with arbitrary coefficients. After taking the asymptotic forms (meaning well away from the resonance), one has:

$$
\begin{aligned}
& \Psi_{e}(x \rightarrow-\infty) \\
& =C_{-} \cos \theta(x) \exp \left(+i I_{p}\left(x, x_{i}\right) / \lambda\right)+D_{-} \sin \theta(x) \exp \left(-i I_{p}\left(x, x_{i}\right) / \lambda\right), \\
& \begin{array}{l}
\Psi_{e}(x \rightarrow+\infty) \\
=C_{+} \sin \theta(x) \exp \left(-i I_{p}\left(x, x_{i}\right) / \lambda\right)+D_{+} \cos \theta(x) \exp \left(+i I_{p}\left(x, x_{i}\right) / \lambda\right) \\
=\left[c_{1} \sin \theta_{i} \exp \left(+i \operatorname{Re} I_{p}\left(x_{i}, x_{0}\right) / \lambda\right)+c_{2} \cos \theta_{i} \exp \left(-i \operatorname{Re} I_{p}\left(x_{i}, x_{0}\right) / \lambda\right)\right] \\
\quad \times \sin \theta(x) \exp \left(-i \operatorname{Re} I_{p}\left(x, x_{0}\right) / \lambda\right) \\
+\left[c_{1}^{*} \cos \theta_{i} \exp \left(-i \operatorname{Re} I_{p}\left(x_{i}, x_{0}\right) / \lambda\right)-c_{2}^{*} \sin \theta_{i} \exp \left(+i \operatorname{Re} I_{p}\left(x_{i}, x_{0}\right) / \lambda\right)\right] \\
\quad \times \cos \theta(x) \exp \left(+i \operatorname{Re} I_{p}\left(x, x_{0}\right) / \lambda\right),
\end{array}
\end{aligned}
$$

where

$$
I_{p}\left(x, x_{i}\right)=\int_{x_{i}}^{x} d x \sqrt{\Lambda+\varphi^{2}(x)} .
$$

Above, $x$ is a general point, $x_{i}$ is the initial point, and $x_{0}$ is a turning point. Now one must solve the problem of connecting the coefficients $C_{-}$and $D_{-}$(known from the initial conditions) to the coefficients $C_{+}$and $D_{+}$(or equivalently $c_{1}$ and $c_{2}$ ). Tedious algebra reveals the solution to the connection problem to be:

$$
\begin{aligned}
& c_{1}=-\frac{\Gamma(-\nu)}{\sqrt{2 \pi}} \Omega^{-i \Omega / 2+\eta / 2}\left(\frac{e^{-i \pi / 2}}{2}\right)^{\nu} \frac{e^{-3 i \pi / 4}}{\sqrt{2}} \exp \left(+\frac{i \Omega}{2}\right) 2 i \sin (\nu \pi) \\
& c_{2}=e^{-i \nu \pi} .
\end{aligned}
$$

Comparison of $\left|\Psi_{e}(x \rightarrow+\infty)\right|^{2}$ above to the general form

$$
P\left(\nu_{e} \rightarrow \nu_{e}\right)=\frac{1}{2}\left[1+\left(1-2 P_{\mathrm{hop}}\right) \cos 2 \theta_{i} \cos 2 \theta_{v}\right]
$$

reveals that:

$$
\begin{aligned}
1-P_{\text {hop }} & =\left|c_{1}\right|^{2}=1-e^{-\Omega \pi}, \\
P_{\text {hop }} & =\left|c_{2}\right|^{2}=e^{-\Omega \pi},
\end{aligned}
$$

where

$$
\Omega=\frac{2 i}{\pi}\left(\frac{\delta m^{2}}{4 E \hbar}\right) \int_{t_{0}}^{t_{0}^{*}} d t \sqrt{\sin ^{2} 2 \theta_{v}+\left(\zeta(t)-\cos 2 \theta_{v}\right)^{2}} .
$$

The dimensions have been restored and $\Lambda$ and $\varphi$ replaced by their definitions; recall that $\zeta(t) \sim$ density and $t_{0}, t_{0}^{*}$ are the turning points (zeros of the integrand). For a linear density, the Landau-Zener result is recovered, i.e.,

$$
\text { linear density : } \Omega=\frac{\delta m^{2}}{4 E \hbar} \frac{\sin ^{2} 2 \theta_{v}}{\cos 2 \theta_{v}}\left|\frac{\dot{\zeta}(t)}{\zeta(t)}\right|_{\text {res }}^{-1} \text {. }
$$


However, any arbitrary monotonic density may be used. For example, for an exponential density,

$$
\text { exponential density : } \Omega=\frac{\delta m^{2}}{4 E \hbar}\left(1-\cos 2 \theta_{v}\right)\left|\frac{\dot{\zeta}(t)}{\zeta(t)}\right|^{-1},
$$

which is the leading term in the exact result. That the approximation is rather good over a wide variety of parameters can be seen in Fig. 2.

\section{Conclusions}

In a semiclassical approximation, one always finds that the wave functions have terms like

$$
\Psi \sim \exp (() / \hbar)
$$

where ( ) indicates a phase integral and various numerical factors. There is an essential singularity in $\Psi$ in the formal limit $\hbar \rightarrow 0$. That is, $\Psi$ does not have a well-defined value in this limit. Different approximations (i.e., different ways of limiting $\hbar \rightarrow 0$ ) therefore give different results. Not all semiclassical approximations are equivalent; other criteria must be used to decide which approach to use. In this level-crossing problem, the best results come from a uniform approximation (valid for all $x$, including the turning points) that explicitly recognizes the supersymmetric nature of the potential. Given the success of similar approximations in the bound-state problem, this is perhaps to be expected.

Even the uniform supersymmetric approximation eventually breaks down. In the original problem (in the figure above, based on the exponential density), we considered only the two turning points closest to the real axis. In general, there are more, further out in the complex plane. The distance of the the turning points from the real axis scales like $|\zeta / \dot{\zeta}|$ (and for the exponential density, integer multiples of this). The analog potential (based on the linear density), only has two turning points. This mismatch in turning-point topologies eventually makes the mapping function multivalued, at which point the approximation breaks down. The problem is worst when the length scale $E \hbar / \delta m^{2}$ becomes large and "sees" further into the complex plane.

This is an interesting practical problem, in part because there is good evidence for matter-enhanced neutrino oscillations. The technique given here is applicable for any monotonic density profile (and not just in the sun), and for a large range of mixing parameters [Balantekin and Beacom (1996)]. In addition, the techniques here should also be useful in a variety of the continuum levelcrossing problems that are ubiquitous in quantum mechanics. As noted, certain kinds of two-level systems are automatically of the supersymmetric form. The techniques of semiclassical SUSY QM for the bound-state problem can be carried over, as shown in our example. 


\section{Acknowledgments}

This work was supported in part by the U.S. National Science Foundation Grant No. PHY-9605140 at the University of Wisconsin, and in part by the University of Wisconsin Research Committee with funds granted by the Wisconsin Alumni Research Foundation.

\section{References}

J.N. Bahcall (1989): Neutrino Astrophysics (Cambridge, New York)

A.B. Balantekin, S.H. Fricke, P.J. Hatchell (1988): "Analytical and Semiclassical Aspects of Matter-Enhanced Neutrino Oscillations," Phys. Rev. D 38, 935

A.B. Balantekin, J.E. Seger (1991): "Dynamical Effects in Pair Production by Electric Fields," Int. J. Mod. Phys. A 6, 695

A.B. Balantekin, J.F. Beacom (1996): "Semiclassical Treatment of MatterEnhanced Neutrino Oscillations for an Arbitrary Density Profile," Phys. Rev. D 54, 6323

A.B. Balantekin (1997): "Selected Topics in Neutrino Astrophysics," astro-ph/9706256, to be published in the Proceedings of the 1997 Jorge Andre Swieca Summer School, Brazil

F. Boehm, P. Vogel (1992): Physics of Massive Neutrinos, 2nd ed. (Cambridge, New York)

A. Comtet, A.D. Bandrauk, D.K. Campbell (1985): "Exactness of Semiclassical Bound State Energies for Supersymmetric Quantum Mechanics," Phys. Lett. B 150, 159

F. Cooper, A. Khare, U. Sukhatme (1995): "Supersymmetry and Quantum Mechanics," Phys. Rep. 251, 267

D. DeLaney, M.M. Nieto (1990): "SUSY-WKB is Neither Exact Nor Never Worse than WKB for all Solvable Potentials," Phys. Lett. B 247, 301

S.H. Fricke, A.B. Balantekin, P.J. Hatchell, T. Uzer (1988): "Uniform Semiclassical Approximation to Supersymmetric Quantum Mechanics," Phys. Rev. A 37, 2797

M. Hruska, W.-Y. Keung, U. Sukhatme (1997): "Accuracy of Semiclassical Methods for Shape-Invariant Potentials," Phys. Rev. A 55, 3345

F. Schwabl (1995): Quantum Mechanics, 2nd ed., Ch. 19 (Springer, Berlin)

A more extensive list of references is given in Balantekin and Beacom (1996). 